\title{
CONVERGENCE OF ORTHOGONAL RATIONAL FUNCTIONS
}

Adhemar Bultheel

Department of Computing Science

K.U. Leuven

Belgium

Pablo Gonzalez-Vera

Department of Mathematical Analysis

University of La Laguna

Tenerife, Spain

Erik Hendriksen

Department of Mathematics

University of Amsterdam

The Vetherlands

Olav . Njåstad

Department of Mathematical Sciences

University of Trondheim - NTH

Norway 


\section{Introduction}

Every finite positive Borel measure $\mu$ on the unit circle gives rise to an orthonormal sequence $\left\{\varphi_{n}\right\}$ of polynomials: Szegö polynomials. The reciprocal polynomials $\left\{\varphi_{n}^{*}\right\}$ are defined by $\varphi_{n}^{*}(z)=z^{n} \overline{\varphi_{n}(1 / \bar{z})}$. The (positive) leading cofficient of $\varphi_{n}(z)$ is called $\kappa_{n}$, it is easily seen that $\kappa_{n}=\varphi_{n}^{*}(0)$. The reflection coefficient $\delta_{n}$ is defined by $\delta_{n}=\varphi_{n}(0) / \kappa_{n}$. The Szegö polynomials and their reciprocals satisfy the coupled recurrence relations

$$
\begin{gathered}
\varphi_{n}(z)=\frac{\kappa_{n}}{\kappa_{n-1}}\left[z \varphi_{n-1}(z)+\delta_{n} \varphi_{n-1}^{*}(z)\right] \\
\varphi_{n}^{*}(z)=\frac{\kappa_{n}}{\kappa_{n-1}}\left[\overline{\delta_{n}} z \varphi_{n-1}(z)+\varphi_{n-1}^{*}(z)\right] \\
\varphi_{0}=\varphi_{0}^{*}=\kappa_{0} .
\end{gathered}
$$

The measure $\mu$ satisfies the Szegö condition $\int_{-\pi}^{\pi} \ln \mu^{\prime}(\theta) d \theta>-\infty$ if and only if $\left\{\kappa_{n}\right\}$ converges to a finite value $\kappa$ and if and only if the series $\sum_{n=1}^{\infty}\left|\delta_{n}\right|^{2}$ converges. When these equivalent conditions are satisfied, the sequence $\left\{\varphi_{n}^{*}\right\}$ converges locally uniformly in the open unit disk to the function

$$
\pi(z)=\frac{1}{\sqrt{2 \pi}} \exp \left[-\frac{1}{4 \pi} \int_{-\pi}^{\pi} \frac{e^{i \theta}+z}{e^{i \theta}-z} \ln \mu^{\prime}(\theta) d \theta\right] .
$$

When the stronger condition $\sum_{n=1}^{\infty}\left|\delta_{n}\right|<\infty$ is satisfied, the sequence $\left\{\varphi_{n}^{*}\right\}$ converges uniformly on the closed unit disk, and the (continuous) limit function does not vanish. For proots and more details concerning these matters, see [12,13,14,15,16,18,23].

Polynomials are rational functions with a pole at infinity only. For some purposes it is useful to work with orthogonal rational functions with prescribed poles outside the unit disk. From a purely mathematical point of view the theory of such orthogonal rational functions was as far as we know initiated by Djrbashian about 1960 (see the survey paper [11]). Independently, partly from an applied point of view, the same constructions were used by Bultheel, Bultheel and Dewilde, Dewilde and Dym about 1980 (see $[1,2,10]$ ). The basic features of a general theory of orthogonal rational functions are set forth in $[3,4,5,6,7,8]$ and in references found there. See also $[20,21]$. The theory is closely connected 
with the Nevanlinna-Pick interpolation problem. See e.g. $[19,20,21]$, and for applications [9].

In [8] the complex of conditions related to the Szegö condition in the rational situation was investigated under the assumption that all the poles are contained in a compact subset (in the extended complex plane $\hat{C}$ ) of the exterior of the unit disk. It was established that (when the Szegö condition is satisfied) subsequences of the reciprocals $\left\{\varphi_{n}^{*}\right\}$ of the orthogonal rational functions $\left\{\varphi_{n}\right\}$ converge locally uniformly in the open unit disk to functions

$$
\pi_{\alpha}(z)=e^{-i \lambda} \frac{\sqrt{1-|\alpha|^{2}}}{\sqrt{2 \pi(1-\bar{\alpha} z)}} \exp \left[-\frac{1}{4 \pi} \int_{-\pi}^{\pi} \frac{e^{i \theta}+z}{e^{i \theta}-z} \ln \mu^{\prime}(\theta) d \theta\right], \quad \lambda \in R .
$$

In particular the whole sequence $\left\{\varphi_{n}^{*}\right\}$ converges to $\pi_{\alpha}$ if the prescribed poles converge to $1 / \bar{\alpha}$.

The main result of this paper is a theorem on uniform convergence of $\left\{\varphi_{n}^{*}\right\}$ on the closed unit disk which generalizes the theorem referred to above for the polynomial case.

\section{Orthogonal functions}

We shal use the notations $T=\{z \in C:|z|=1\}, D=\{z \in C:|z|<1\}, E=\{z \in$ $C:|z|>1\}$. The substar conjugate $f$, of a function $f$ is defined by

$$
f_{*}(z)=\overline{f(1 / \bar{z})} .
$$

Let $\mu$ be a finite (positive) Borel measure on $(-\pi, \pi]$. An inner product is defined by

$$
<f, g\rangle=\int_{-\pi}^{\pi} f\left(e^{i \theta}\right) g \overline{\left(e^{i \theta}\right)} d \mu(\theta)=\int_{-\pi}^{\pi} f\left(e^{i \theta}\right) g \cdot\left(e^{i \theta}\right) d \mu(\theta) .
$$

Let $\left\{\alpha_{n}\right\}$ be an arbitrary sequence of (not necessarily distinct) points in $D$. It is sometimes convenient to use the notation $\alpha_{0}=0$. The Blaschke factors $\zeta_{n}$ are defined by

$$
\zeta_{n}(z)=\tau_{n} \frac{\left(\alpha_{n}-z\right)}{\left(1-\overline{\alpha_{n}} z\right)}, \text { where } \tau_{n}=\frac{\overline{\alpha_{n}}}{\left|\alpha_{n}\right|}, n=1,2, \ldots .
$$


(By convention, $\tau_{n}=-1$ when $\alpha_{n}=0$.) The Blaschke products $B_{n}$ are defined by

$$
B_{0}(z)=1, \quad B_{n}(z)=\prod_{k=1}^{n} \zeta_{k}(z) \text { for } n=1,2, \ldots .
$$

We define the spaces $\mathcal{L}_{n}$ by

$$
\mathcal{L}_{n}=\operatorname{Span}\left\{B_{m}: m=0,1, \ldots, n\right\} .
$$

The elements of $\mathcal{L}_{n}$ are exactly the functions that may be written in the form

$$
L(z)=\frac{p_{n}(z)}{\pi_{n}(z)}
$$

where

$$
\pi_{n}(z)=\prod_{k=1}^{n}\left(1-\overline{\alpha_{k}} z\right)
$$

and $p_{n} \in \Pi_{n}$ (the space of polynomials of degree at most $n$ ). In particular $\mathcal{L}_{n}=\prod_{n}$ when $\alpha_{n}=0$ for all $n$.

For $f \in \mathcal{L}_{n} \backslash \mathcal{L}_{n-1}$ we define the superstar conjugate $f^{*}$ by

$$
f^{*}(z)=B_{n}(z) f_{*}(z) \text {. }
$$

Observe that $f^{*} \in \mathcal{L}_{n}$.

Let the sequence $\left\{\Phi_{n}: n=0,1,2, \ldots\right\}$ be obtained by orthogonalization of the sequence $\left\{B_{n}: n=0,1,2, \ldots\right\}$ with respect to the inner product (2.2). Each $\Phi_{n}$ has a decomposition

$$
\Phi_{n}(z)=\sum_{k=0}^{n} b_{k}^{(n)} B_{k}(z)
$$

By calculating $\Phi_{n}^{*}(z)$ and substituting $\alpha_{n}$ for $z$ we see that

$$
\overline{\Phi_{n}^{*}\left(\alpha_{n}\right)}=b_{n}^{(n)} .
$$

We shall reserve the notation $\Phi_{n}$ for the monic functions, i.e. those for which $b_{n}^{(n)}=1$. We set

$$
\kappa_{n}=\left\langle\Phi_{n}, \Phi_{n}>-\frac{1}{2}\right.
$$


and denote by $\varphi_{n}$ the normalized functions:

$$
\varphi_{n}(z)=\kappa_{n} \Phi_{n}(z) .
$$

We thus have

$$
\begin{aligned}
& \left\langle\varphi_{n}, \varphi_{n}\right\rangle=\left\langle\varphi_{n}^{*}, \varphi_{n}^{*}\right\rangle=1 \\
& \varphi_{n}^{*}\left(\alpha_{n}\right)=\kappa_{n}, \Phi_{n}^{*}\left(\alpha_{n}\right)=1 .
\end{aligned}
$$

It can be shown that $\varphi_{n}^{*}$ solves the extremum problem

$$
\max \left\{\left|f\left(\alpha_{n}\right)\right|: f \in \mathcal{L}_{n},\langle f, f>=1\} .\right.
$$

The sequence $\left\{\mathcal{L}_{n}\right\}$ is nested, i.e. $\mathcal{L}_{n} \subset \mathcal{L}_{n+1}$. It follows that the sequence $\left\{\kappa_{n}\right\}=$ $\left\{\varphi_{n}^{*}\left(\alpha_{n}\right)\right\}$ is non-decreasing if $\alpha_{n}=\alpha$ for all $n$. This monotonicity property of $\left\{\kappa_{n}\right\}$ does not follow in general.

The functions $\varphi_{n}$ have all their zeros in $D$, while the functions $\varphi_{n}^{*}$ have all their zeros in $E$.

The sequences $\left\{\varphi_{n}\right\},\left\{\varphi_{n}^{*}\right\}$ satisty the following coupled recurrence relations:

$$
\begin{gathered}
\varphi_{n}(z)=\frac{\kappa_{n}}{\kappa_{n-1}}\left[\varepsilon_{n} \frac{z-\alpha_{n-1}}{1-\overline{\alpha_{n}} z} \varphi_{n-1}(z)+\delta_{n} \frac{1-\overline{\alpha_{n-1}} z}{1-\overline{\alpha_{n}} z} \varphi_{n-1}^{*}(z)\right] \\
n=1,2, \ldots \\
\varphi_{n}^{*}(z)=-\tau_{n} \frac{\kappa_{n}}{\kappa_{n-1}}\left[\overline{\delta_{n}} \frac{z-\alpha_{n-1}}{1-\overline{\alpha_{n}} z} \varphi_{n-1}(z)+\frac{\left.1-\overline{\delta_{n}} \frac{1-\overline{\alpha_{n-1}} z}{1-\overline{\alpha_{n}} z} \rho_{n-1}^{*}(z)\right]}{n=1,2, \ldots}\right. \\
\alpha_{0}=0, \varphi_{0}=\kappa_{0}, \varphi_{0}^{*}=\kappa_{0} .
\end{gathered}
$$

The recurrence coefficients $\delta_{n}, \varepsilon_{n}$ are given by

$$
\delta_{n}=\frac{\left(1-\alpha_{n-1} \overline{\alpha_{n}}\right)}{\left(1-\left|\alpha_{n-1}\right|^{2}\right)} \frac{\varphi_{n}\left(\alpha_{n-1}\right)}{\kappa_{n}}
$$




$$
\varepsilon_{n}=-\tau_{n} \frac{\left(1-\overline{\alpha_{n-1}} \alpha_{n}\right) \overline{\varphi_{n}^{*}\left(\alpha_{n-1}\right)}}{\left(1-\left|\alpha_{n-1}\right|^{2}\right) \kappa_{n}} .
$$

The coefficient $\varepsilon_{n}$ is always different from zero. (Follows e.g. by substituting $z=\alpha_{n-1}$ in (2.17), taking into account that $\varphi_{n}^{*}$ has no zeros in D.) Furthermore $\left|\delta_{n}\right|<\left|\varepsilon_{n}\right|$.

The sequences $\left\{\kappa_{n}\right\},\left\{\delta_{n}\right\},\left\{\varepsilon_{n}\right\}$ are connected through the formula

$$
\frac{\kappa_{0}^{2}}{\kappa_{n}^{2}}\left(1-\left|\alpha_{n}\right|^{2}\right)=\prod_{m=1}^{n}\left[\left|\varepsilon_{m}\right|^{2}-\left|\delta_{m}\right|^{2}\right] \text {. }
$$

Clearly $\left\{\frac{\kappa_{n}}{\sqrt{1-\left|\alpha_{n}\right|^{2}}}\right\}$ converges to a finite value if and only if the infinite product

$\prod_{m=1}^{\infty}\left[\left|\varepsilon_{m}\right|^{2}-\left|\delta_{m}\right|^{2}\right]$ converges (to a finite value different from zero).

For more exhaustive treatments of the concepts and results referred to, see $[3,4,5,6,7,8]$.

\section{Locally uniform convergence}

We shall in the rest of this paper assume that the sequence $\left\{\alpha_{n}\right\}$ converges to a limit $\alpha$ in $D$ :

$$
\lim _{n \rightarrow \infty} \alpha_{n}=\alpha, \alpha \in D
$$

(More general results can be obtained by only assuming that ail $\alpha_{n}$ are contained in a compact subset of $D$, by considering accumulation points and convergent subsequences. Cf. [3].)

We recall that the measure $\mu$ is said to satisfy the Szegö condition if

$$
\int_{-\pi}^{\pi} \ln \mu^{\prime}(\theta) d \theta>-\infty \text {. }
$$

(Here $\mu^{\prime}(\theta)$ denotes the derivative of $\mu$ - and of the absolutely continuous part of $\mu$ - with respect to Lebesgue measure.)

We now state as a theorem some basic results connecting the Szegö condition with behavior of the sequences $\left\{\kappa_{n}\right\},\left\{\delta_{n}\right\},\left\{\varepsilon_{n}\right\}$ : 
Theorem 3.1 The following conditions are equivalent when (3.1) is satisfied: $\left\{\kappa_{n}\right\}$ converges (to a finite value different from zero). $\prod_{m=1}^{\infty}\left[\left|\varepsilon_{m}\right|^{2}-\left|\delta_{m}\right|^{2}\right]$ converges (to a finite value different from zero).

Proof: Follows from [8, Theorem 6.10].

The Szegö spectral factor $\sigma_{\mu}$ is defined by

$$
\sigma_{\mu}(z)=\sqrt{2 \pi} \exp \left[\frac{1}{4 \pi} \int_{-\pi}^{\pi} \frac{e^{i \theta}+z}{e^{i \theta}-z} \ln \mu^{\prime}(\theta) d \theta\right] .
$$

(We may set $\sigma_{\mu} \equiv 0$ when the Szegö condition (3.2) is not satisfied.)

We state a main result on convergence of the sequence $\left\{\varphi_{n}^{*}\right\}$.

Theorem 3.2 Assume that (3.1) and (3.2) (hence the conditions (A), (B), (C)) are satisfied. Then $\left\{\varphi_{n}^{*}\right\}$ converges locally uniformly in $D$ to a function

$$
\pi_{\alpha}(z)=e^{-i \lambda} \frac{\sqrt{1-|\alpha|^{2}}}{(1-\bar{\alpha} z) \sigma_{\mu}(z)}, \lambda \in R .
$$

Proof: Follows from [8, Theorem 6.10 and Theorem 6.12].

Corollary 3.3 Assume that

$$
\alpha_{n}=\alpha \text { for all } n
$$

and that

$$
\sum_{n=1}^{\infty}\left|\hat{o}_{n}\right|^{2}<\infty
$$

Then $\left\{\boldsymbol{\varphi}_{n}^{*}\right\}$ converges locally uniformly in $D$ to the function $\pi_{\alpha}$ given by (3.4).

Proof: It follows from (2.14), (2.20) and (3.5) that in this case $\left|\varepsilon_{n}\right|=1$, and so (3.6) is equivalent to condition (B). The result then follows from Theorem 3.1 and Theorem 3.2. 
For the sake of completeness we also include the following result.

Theorem 3.4 Assume that (3.1) and (3.2) (hence the conditions (A), (B), (C)) are satisfied. Then the reproducing kernels

$$
k_{n}(z, w)=\sum_{m=0}^{n} \varphi_{m}(z) \overline{\varphi_{m}(w)}
$$

converge locally uniformly for $z, w \in D$ to the function

$$
s(z, w)=\frac{1}{(1-z \bar{w}) \sigma_{\mu}(z) \overline{\sigma_{\mu}(w)}},
$$

i.e.

$$
\sum_{m=0}^{\infty} \varphi_{m}(z) \overline{\varphi_{m}(w)}=\frac{1}{(1-z \bar{w}) \sigma_{\mu}(z) \overline{\sigma_{\mu}(w)}} .
$$

Proof: Follows from [8, Theorem 6.14].

\section{Uniform convergence}

We recail that we shall also in this section assume that (3.1) is satisfied. We shail prove stronger convergence results for $\left\{\varphi_{n}^{*}\right\}$ under a stronger condition than (3.2). This condition is

$$
\sum_{m=1}^{\infty}\left[\left|1+\tau_{m} \overline{\xi_{m}}\right|+\left|\delta_{m}\right|\right]<\infty .
$$

We shail first establish that this condition is in tact stronger than (3.2).

Proposition 4.1 Assume that (4.1) is satisfied. Then the product

$$
\prod_{m=1}^{\infty}\left[\left|\varepsilon_{m}\right|^{2}-\left|\delta_{m}\right|^{2}\right]
$$


converges absolutely.

Proof: First note that

$$
|| \varepsilon_{m}|-1| \leq\left|1+\tau_{m} \bar{\Xi}_{m}\right|
$$

and

$$
\left.|| \varepsilon_{m}\right|^{2}-1|\leq|\left|\varepsilon_{m}\right|-1 \mid\left(\left|\varepsilon_{m}\right|+1\right) .
$$

It follows from (4.1) that

$$
\left|\varepsilon_{m}\right|+1 \leq M<\infty, \sum_{m=1}^{\infty}\left|\delta_{m}\right|^{2}<\infty .
$$

Together $(4.1) \cdot(4.4)$ give

$$
\left.\sum_{m=1}^{\infty}\left|\left(\left|\varepsilon_{m}\right|^{2}-1\right)-\right| \delta_{m}\right|^{2} \mid<\infty .
$$

From a basic convergence criterion for infinite products (see e.g. [17]) we then conclude that the product

$$
\prod_{m=1}^{\infty}\left[\left|\varepsilon_{m}\right|^{2}-\left|\delta_{m}\right|^{2}\right]=\prod_{m=1}^{\infty}\left[1+\left(\left|\varepsilon_{m}\right|^{2}-1-\left|\delta_{m}\right|^{2}\right)\right]
$$

converges absolutely.

Corollary 4.2 Assume that (3.1) and (4.1) are satisfied. Then the conditions (A), (B), (C) are satisfied.

Proof: Follows from Theorem 3.1 and Proposition 4.1.

We now prove our main result.

Theorem 4.3 Assume that (3.1) and (4.1) are satisfied. Then $\left\{\varphi_{n}^{*}\right\}$ converges uniformly on $D \cup T$. It follows that $\pi_{\alpha}$ has a continuous extension to $D \cup T$. Furthermore

$$
\min _{z \in D \cup T}\left|\pi_{x}(z)\right|>0
$$


(where $\pi_{\alpha}$ denotes the extended function).

Proof: From the recurrence relation (2.17) follows

$$
\begin{aligned}
& \frac{\varphi_{m}^{*}(z)}{\varphi_{m-1}^{*}(z)}=-\tau_{m} \frac{\kappa_{m}\left(1-\overline{\alpha_{m-1}} z\right)}{\kappa_{m-1}\left(1-\overline{\alpha_{m}} z\right)}\left[\overline{\varepsilon_{m}}+\overline{\delta_{m}} \frac{z-\alpha_{m-1}}{1-\overline{\alpha_{m-1}} z} \frac{\varphi_{m-1}(z)}{\varphi_{m-1}^{*}(z)}\right], \\
& m=1,2, \ldots .
\end{aligned}
$$

Multiplication of (4.9) for $m=1,2, \ldots n$ together with (2.18) gives

$$
\varphi_{n}^{*}(z)=\varphi_{0}^{*}(z) \prod_{m=1}^{n} \frac{\left(1-\overline{\alpha_{m-1}} z\right)}{\left(1-\overline{\alpha_{m}} z\right)} \frac{\kappa_{m}}{\kappa_{m-1}}\left(-\tau_{m}\right)\left[\overline{\varepsilon_{m}}+\overline{\delta_{m}} \frac{\left(z-\alpha_{m-1}\right) \varphi_{m-1}(z)}{\left(1-\overline{\alpha_{m-1}} z\right) \varphi_{m-1}^{*}(z)}\right]
$$

hence

$$
\varphi_{n}^{*}(z)=\frac{\kappa_{n}}{1-\bar{\alpha}_{n} z} \prod_{m=1}^{n}\left(-\tau_{m}\right)\left[\overline{\varepsilon_{m}}+\bar{\delta}_{m} \frac{\left(z-\alpha_{m-1}\right) \varphi_{m-1}(z)}{\left(1-\overline{\alpha_{m-1}} z\right) \varphi_{m-1}^{*}(z)}\right]
$$

We note that $\frac{\kappa_{n}}{1-\overline{\alpha_{n}} z}$ converges to $\frac{\kappa}{1-\bar{\alpha} z}\left(\right.$ with $\left.\kappa=\lim _{n} \kappa_{n}\right)$ uniformly on $D \cup T$. We may write the product in (4.11) as

$$
\begin{array}{r}
\prod_{m=1}^{n}\left(-\tau_{m}\right)\left[\overline{\varepsilon_{m}}+\overline{\delta_{m}} \frac{\left(z-\alpha_{m-1}\right) \varphi_{m-1}(z)}{\left(1-\overline{\alpha_{m-1}} z\right) \varphi_{m-1}^{*}(z)}\right] \\
=\prod_{m=1}^{n}\left\{1+\left[\left(-\tau_{m} \overline{\bar{\xi}_{m}}-1\right)-\tau_{m} \overline{\delta_{m}} \frac{\left(z-\alpha_{m-1}\right) \varphi_{m-1}(z)}{\left(1-\overline{\alpha_{m-1}} z\right) \varphi_{m-1}^{*}(z)}\right]\right\} .
\end{array}
$$

The function $\frac{\left(z-\alpha_{m-1}\right) \varphi_{m-1}(z)}{\left(1-\overline{\alpha_{m-1}} z\right) \varphi_{m-1}^{*}(z)}$ is analytic on $D \cup T$ (since $\alpha_{m-1} \in D$ and the zeros of $\varphi_{m-1}^{*}(z)$ are in $\left.E\right)$, and

$$
\left|\frac{\left(z-\alpha_{m-1}\right) \varphi_{m-1}(z)}{\left(1-\overline{\alpha_{m-1}} z\right) \varphi_{m-1}^{\cdot}(z)}\right|=1 \text { for } z \in T .
$$

Consequently

$$
\left|\frac{\left(z-\alpha_{m-1}\right) \varphi_{m-1}(z)}{\left(1-\overline{\alpha_{m-1}} z\right) \varphi_{m-1}^{*}(z)}\right| \leq 1 \text { for } z \in D \cup T \text {. }
$$

hence

$$
\begin{aligned}
& \left|\left(-\tau_{m} \overline{\bar{s}_{m}}-1\right)-\tau_{m} \overline{\delta_{m}} \frac{\left(z-\alpha_{m-1}\right) \varphi_{m-1}(z)}{\left(1-\overline{\alpha_{m-1} z}\right) \varphi_{m-1}^{*}(z)}\right| \\
& \quad \leq\left|1+\tau_{m} \overline{\bar{s}_{m}}\right|+\left|\delta_{m}\right| \text { for } z \in D \cup T .
\end{aligned}
$$


The assumption (4.1) together with (4.15) implies that the product in (4.11) and hence $\left\{\varphi_{n}^{*}\right\}$ converges uniformly on $D \cup T$ to a continuous function, which on $D$ coincides with $\pi_{\alpha}$. The limit function is different from zero since all the factors in the infinite product are different from zero and a convergent infinite product is zero only if one of the factors are zero.

Remark It follows from (4.1) that $\left|1+\tau_{m} \overline{\bar{s}_{m}}\right|+\left|\delta_{n}\right|<1$ for sufficiently large $m$, say $m \geq M$. Hence for $m \geq M$ :

$$
\begin{aligned}
\mid 1+\left(-\tau_{m} \overline{\bar{\xi}_{m}}-1\right) & -\tau_{m} \overline{\delta_{m}} \frac{\left(z-\alpha_{m-1}\right) \varphi_{m-1}(z)}{\left(1-\overline{\alpha_{m-1}} z\right) \varphi_{m-1}^{*}(z)} \mid \\
& \geq\left|1-\left[\left|1+\tau_{m} \overline{\bar{\xi}_{m}}\right|+\left|\delta_{m}\right|\right]\right|
\end{aligned}
$$

which means

$$
\left|\left(-\tau_{m}\right)\left[\overline{\varepsilon_{m}}+\overline{\delta_{m}} \frac{\left(z-\alpha_{m-1}\right) \varphi_{m-1}(z)}{\left(1-\overline{\alpha_{m-1}} z\right) \varphi_{m-1}^{*}(z)}\right]\right| \geq\left|1-\left[\left|1+\tau_{m} \overline{\varepsilon_{m}}\right|+\left|\delta_{m}\right|\right]\right| .
$$

Consequently

$$
\begin{array}{r}
\left|\prod_{m=. h}^{\infty}\left(-\tau_{m}\right)\left[\overline{\varepsilon_{m}}+\overline{\delta_{m}} \frac{\left(z-\alpha_{m-1}\right) \varphi_{m-1}(z)}{\left(1-\overline{\alpha_{m-1}} z\right) \varphi_{m-1}^{*}(z)}\right]\right| \\
\geq \prod_{m=. h}^{\infty}\left|\left\{1-\left[\left|1+\tau_{m} \overline{\bar{\varepsilon}_{m}}\right|+\left|\delta_{m}\right|\right]\right\}\right|
\end{array}
$$

for $z \in D \cup T$. Thus $($ recall $(4.11))$

$$
\begin{array}{r}
\lim _{n \rightarrow \infty}\left|\varphi_{n}^{*}(z)\right| \geq \frac{\zeta}{1-|\alpha|}\left|\prod_{m=1}^{M-1}\left(-\tau_{m}\right)\left[\overline{\varepsilon_{m}}+\overline{\delta_{m}} \frac{\left(z-\alpha_{m-1}\right)\left(\varphi_{m-1}(z)\right.}{\left(1-\overline{\alpha_{m-1}} z\right) \varphi_{m-1}^{*}(z)}\right]\right| \prod_{m=M}^{\infty} \\
\left\{1-\left[\left|1+\tau_{m} \overline{\xi_{m}}\right|+\left|\delta_{m}\right|\right]\right\}
\end{array}
$$

If $\left|1+\tau_{m} \overline{\xi_{m}}\right|+\left|\delta_{m}\right|<1$ for all $m$, then

$$
\lim _{n \rightarrow \infty}\left|\varphi_{n}^{*}(z)\right| \geq \frac{<}{1-|\alpha|} \prod_{m=1}^{\infty}\left\{1-\left[\left|1+\tau_{m} \frac{\overline{\xi_{m}}}{\bar{m}_{m}}+\right| \delta_{m} \mid\right]\right\} .
$$

Corollary 4.4 Assume that

$$
\alpha_{n}=\alpha \text { for all } n
$$


and that

$$
\sum_{n=1}^{\infty}\left|\delta_{n}\right|<\infty .
$$

Then $\left\{\varphi_{n}^{*}\right\}$ converges uniformly on $D \cup T$, and

$$
\min _{z \in D \cup T}\left|\pi_{\alpha}(z)\right|>0
$$

(where $\pi_{\alpha}$ denotes the continuous limit function on $D \cup T$ ).

Proof: It follows from (2.14), (2.20) and (4.21) that in this situation $\varepsilon_{m}=-\tau_{m}$, hence $\tau_{m} \overline{\xi_{m}}=-\left|\tau_{m}\right|^{2}=-1$. Condition (4.1) then reduces to (4.20), and the result follows from Theorem 4.3 .

\section{REFERENCES}

1. A. Buitheel, On a special Laurent-Hermite interpolation problem, Numerische Methoden der Approximationstheorie 6 (Ed. L. Collatz, G. Meinardus, H. Werner; Birkhäuser, Basel 1981) 63-70.

2. A. Bultheel and P. Dewilde, Orthogonal functions related to the Nevanlinna-Pick problem, Mathematical Theory of Networks and Systems, Proceedings MTNS Conference, Delft, The Nerherlands (Ed. P. Dewilde; Western Periodicals, North Hollywood, 1979) 207-211.

3. A. Bultheel, P. Gonzalez-Vera, E. Hendriksen and O. Njåstad, A Szegö theory for rational functions, Technical Report TW-131, K.U. Leuven, Dept. of Computer Science, May 1990.

4. A. Bultheel, P. Gonzalez-Vera. E. Hendriksen and O. Njåstad, The computation of orthogonal rational functions and their interpolating properties, Numerical Algorithms, 2 (1992) 85-114. 
5. A. Bultheel, P. Gonzalez-Vera, E. Hendriksen and O. Njåstad, Moment problems and orthogonal functions, J. Comp. Appl. Math., to appear.

6. A. Bultheel, P. Gonzalez-Vera, E. Hendriksen and O. Njảstad, Orthogonal rational functions with poles on the unit circle, J. Math. Anal. Appl., to appear.

7. A. Bultheel, P. Gonzalez-Vera, E. Hendriksen and O. Njăstad, Recurrence relations for orthogonal functions, Continued Fractions and Orthogonal Functions (Ed. S.C. Cooper and W.J. Thron; Marcel Dekker), to appear.

8. A. Bultheel, P. Gonzalez-Vera, E. Hendriksen and O. Njåstad, Asymptotics for orthogonal rational functions, submitted.

9. P. Delsarte, Y. Genin and Y. Kamp, On the role of the Nevanlinna-Pick problem in circuit and system theory, Int. J. Circuit Th. Appl. 9 (1981) 177-187.

10. P. Dewilde and H. Dym, Schur recursion, error formulas, and convergence of rational estimators for stationary stochastic sequences, IEEE Trans. on Information Theory, IT 27 (1981) 446-461.

11. M..M. Djrbashian, A survey on the theory of orthogonal systems and some open problems, Orthogonal Polynomials: Theory and Practice (Ed. P. Nevai; Kluwer, Dordrecht-Boston-Londion 1990) 135-146.

12. Y.L. Geronimus, Polynomials orthogonal on a circle and their applications, Amer. Math. Soc. Trans. 104 (1954).

13. Y.L. Geronimus, Orthogonal polynomials, Consuitants Bureau, New York, 1961.

14. U. Grenander and G. Szegö, Toeplitz forms and their applications (Chelsea, Berkley, 1958).

15. W.B. Jones, O. Njảstad and W.J. Thron, .Voment theory, orthogonal polynomials, quadrature, and continued fractions associated with the unit circle, Buil. Lond. Math. Soc. 21 (1989) 113-152.

16. W.B. Jones and O. Njåstad, Applications of Szegö polynomials to digital signal processing, Rocky Mountain J. Math. 21 (1991) 387-436. 
17. K. Knopp, Theorie und Anwendung der unendlichen Reihen, Springer Verlag, BerlinHeidelberg $194 i$.

18. H.J. Landau (Ed.), Moments in Mathematics, Proc. Sympos. Applied Math. 37, American Mathematical Society (Providence 1987).

19. R. Nevanlinna, Über beschränkte Functionen die in gegebenen Punkten vorgeschriebene Werte annehmen, Ann. Acad. Sci. Fenn. Sec. A, 13 (1919) No. 1.

20. K. Pan, Asymptotics for orthogonal systems of rational functions on the unit circle with a given set of poles, Department of Mathematics, University of California, Riverside, Preprint (1991).

21. K. Pan, On characterization theorems for measures associated with orthogonal systems of rational functions on the unit circle, J. Approximation Theory 70 (1992) $265-272$.

22. G. Pick, Über die Beschränkungen analytischer Funktionen welche durch vorgegebene Functionswerte bewirkt werden, Math. Ana. 77 (1916) $7-23$.

23. G. Szegö, Orthogonal polynomials, Amer. Math. Soc. Coll. Publ. XXIII, 4.ed. Providence, R.I., 1975. 